\section{Isolating Vibration on Light Microscopes?}

The following are answers to the above question as recently provided on the Microscopy Listserver:

- A slab of cement, marble, etc. about 24 inches square and 2 inches thick for mass. The heavier the better. Your local paving store will oblige. Four tennis balls under the slab for air springs. OR the inner tube of a tire with the valve relocated to the outside for easy re-inflation is a more compliant isolation spring

- - Mel Dickson

- The Zeiss confocal as some sort of airbag/tyre system for vibration isolation, like the suggested tractor inner tubes. We have used a bicycle inner tube about $3 / 4$ filled with water under a heavy terrazzo (fake marble) slab - seems to work OK

\section{- - Rosemary White, Monash University, Australia}

- Tennis balls under a wooden frame, on which the LM sits. We have had 6 under a large Zeiss Universal for years and we are right next to a busy road on the 2nd floor. Works like a dream!

- Diana van Driel, Sydney University, Australia

- Try A.Q. Sorbothane feet - A set of four hockey puk style "rubber" feet that should cut vibration on most scopes. If not, try a Kinetica air table at $\$ 3,500.00$. Sorbotane or other vibration absorbing products can be found in any audiophile magazine - a big mail order company that would have it is Audio Advisor at (800)942-0220. Four Sorbothane big feet should run $\$ 50.00$ and for heavier scopes there are Sims Navacom silencers for about $\$ 75.00$. Or try the Needle Doctor at (800)229-0644.

\section{- . - Scott E. Berman, Advanced Imaging Concepts}

\section{Diffraction Simulation} \& Analysis Software for the Macintosh ${ }^{\otimes}$

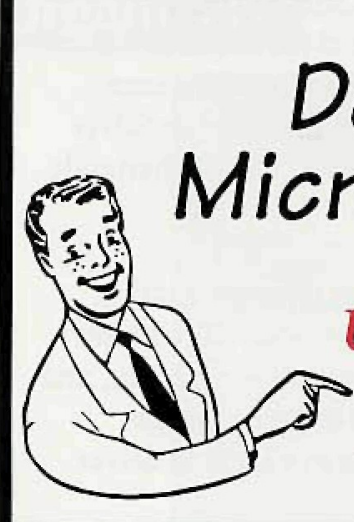

http://www.Rt66.com/ virtlabs/

Upgrades / Demos Now Available via the Internet!

\author{
TEM $\cdot$ SEM $\cdot \mathrm{X}$-RAY \\ $\mathrm{SAD} \cdot \mathrm{CBED} \cdot \mathrm{BEKP} \cdot \mathrm{HOLZ}$ Lines \\ EDD Search - Space Group Tables \\ Kikuchi Maps - Ring Patterns \\ Stereographic Projections \\ Much More?
}

VIRTUAL LABORATORIES

tel: 505-828-1640 - fax: 505-822-9759

Desktop Microscopist is a trademark of Virtual Laboratorles. All other tradernarks are property of their respective owners.

\section{Field Emission Basics: The Water Bucket Analogy \\ Doug Rathkey, FEI Company, Hillsboro, OR}

This article uses an interesting analogy to define and compare the three basic mechanisms of electron emission: photoemission, thermionic emission, and field emission.

The Fermi Level and Three Emission Mechanisms

In the water bucket analogy (Figure 1), the water level in a bucket represents the Fermi level - the highest occupied energy level in the cathode material. The work function is the energy required to get the "water droplets" (electrons) from the top of the liquid out of the bucket and over the side (i.e., the distance equivalent to the potential energy barrier).

In photoemission, the energy of a photon can remove an electron at the Fermi level from the cathode material and can impart enough kinetic energy of travel to allow it to escape from the bucket (Figure 1a). In thermionic emission, heat provides the energy to boil the electrons off and out of the bucket (Figure 1b). Finally, in field emission, a high electric field can thin the side of the bucket enough so that the electrons can tunnel right through it (Figure 1c). There are two types of field emission: cold field emission (CFE) and Schottky emission (SE).

Figure 2 presents the corresponding energy level diagram for thermionic emission (Figure 1b). Theoretically, thermionic emission has no electric field, and therefore, has an infinitely thick potential energy barrier (in our analogy. this means that the wall of the water bucket would be infinitely thick). In practice, an electric field must be applied to allow the electrons to escape from the surface of the cathode material.

Figure 3 presents the energy level diagrams for CFE and SE. Both have similar energy spreads, but their energy distributions are mirror images. In CFE, electrons tunnel from various energies below the Fermi level, while with $\mathrm{SE}$, thermally excited electrons escape over a field-lowered potential energy barrier. In commercial focusing columns, submicron electron beams are produced using two of the three emission mechanisms: thermionic emission (tungsten and $\mathrm{LaB}_{6}$ emitters) and both CFE (pointed tungsten emitters) and SE (SE cathodes).

The CFE and SE sources provide a higher brightness electron source than conventional thermionic sources. Brightness is defined as the on-axis current-per-unit solid angle, divided by the virtual area of the emitting source. This means an increase in current density for nanometer focused beam sizes - particularly for low beam voltage applications where source brightness is a key factor. CFE and SE sources are being used for applications such as electron beam lithography, low voltage SEMs, and some surface analysis equipment, for which small focus beam size and high current density are key requirements. Thermionic sources, such as $\mathrm{LaB}_{6}$, and tungsten filaments, continue to be used in focusing columns that support traditional SEM and surface analytical applications.

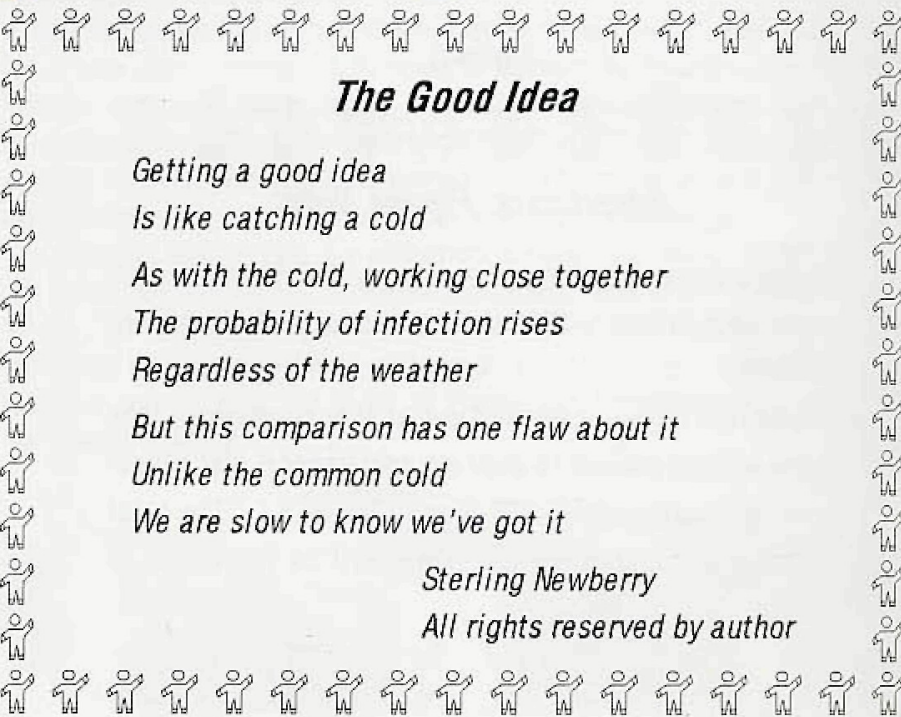

